\title{
Optimization in 3D Scene Acquisition with Two Mobile Robots
}

\author{
Nera González, José M. Sebastián, and Jorge Artieda \\ DISAM, Departamento de Automática, Electrónica e Informática Industrial. Universidad \\ Politécnica de Madrid. José Gutierrez Abascal, 2, \\ 28006 Madrid, Spain \\ neragregmail.com, jsebas@etsii.upm.es, jatrigueroseargongra.com
}

\begin{abstract}
This article presents a method for cooperative reconstruction of three-dimensional scenes using multiple views. Although the use of multiple views is one of the most applied in the 3D scenes interpretation, its use with a single mobile robot does not guarantee the perfect localization of the environment, due to odometry errors. Also, the reconstruction of dynamic environments is not allowed for a single robot, which takes views in different instants. This work proposes a system formed by multiple robots to get a cooperative reconstruction of the scene. The robots decide the best strategy to acquire the image to get the best reconstruction using an objective function defined. In this function, mainly are considered uncertainty of the reconstruction and view points. The result of this optimization is the next position of the robots.
\end{abstract}

Keywords: Uncertainty, Minimization, Reconstruction, Vision, Occlusion.

\section{Introduction}

The objective of this work is to give a navigation strategy for a system of cooperative robots in an unknown environment. The trajectory calculated minimizes the uncertainty in the three-dimensional reconstruction. Robots are terrestrial vehicles which explores a scene using computer vision. Robots should explore the environment minimizing the uncertainty of the $3 \mathrm{D}$ reconstruction. The use of multiple robots for reconstruction leads to a cooperative navigation scheme. In this algorithm robots alternates in its motion to attain a position from where the uncertainty of the reconstruction is reduced.

Uncertainty in three-dimensional reconstruction form visual information is a measurement of the precision of the reconstruction. Given the probability distribution of the error on the input, visual information, the objective is to estimate the probability distribution of the error on the reconstruction. This is done using error propagation models. Among the variables involved in the estimation of the uncertainty is the relative position between robots and the object observed. This leads to the idea of the generation of trajectories that allow a better reconstruction.

\subsection{State of the Art}

There are few works about uncertainty reduction based navigation and mapping with collaborative robots. The algorithms to perform this task are presented for different 
applications but none of the works presents a specific approach for this problem. In this section, we will describe significant developments in uncertainty estimation for visual inspection systems, occlusion, exploration and collaborative systems.

Uncertainty estimation is a key problem for visual inspection systems. These systems need to know the quality of their reconstruction to evaluate the inspected parts. These systems frequently use stereo heads to reconstruct the $3 \mathrm{D}$ model. This is the main difference between these systems and the system presented in this article, because the relative position is an important parameter for the resultant uncertainty in the reconstruction. Examples of these studies are [1]. One study which considers the parameters of relative position between the cameras is [2]. A full 3D reconstruction model which considers the resultant uncertainty is [3], however extrinsic parameters remain fixed. The work presented in [4] considers extrinsic parameters but the relative position between cameras remains fixed. This work also uses optimization techniques to find the position of the stereo head.

Another difference between inspection systems and the configuration presented here is that inspection systems are implemented in restricted environments where occlusion is rarely a problem. The main problem in occlusion considerations is its intensive computational cost. There are a lot of works related with the treatment of occlusion and the reduction of its computational cost such as [5] and [6]. The work presented in [7] deals with an algorithm for estimating the next optimal position for reconstruction taking in account the occlusion. The approach of this algorithm uses voxels. This approach has problems when mapping big environments given the high memory requirements. [8] computes the next position of a laser sensor for exploring the occluded areas but it lacks of the uncertainty approximation.

An important characteristic for the implementation of this kind of navigation algorithm is considering exploration movements. [7] and [8] optimize the position of the cameras to explore new areas while avoiding the occlusions. [9] and [10] uses a cell division of the space to characterize the unexplored areas; the problem of this approach is the application to large environments. [11] uses heuristic methods to get a direction of the movement for exploration.

A natural extension of exploration algorithm is the use of groups of robots to perform the task faster. The use of multiple robots in visual reconstruction with cameras has the advantage of allowing direct reconstruction of the scene. [13] use a team of robot to reconstruct a given objective. The position of the robot is evaluated using a function of the distance and angle to the objective. [14] localize a group of robots using the observed characteristics on the scene. [15] uses a group of robots to estimate the location of mobile characteristics on the scene. [16] and [17] analyzes the problem of exploration and reconstruction using large teams of robots.

There are a lot of developments that provide the tools for the algorithm proposed. On the other hand there is not an approach which completes the problem of getting the next position of a robot which reduces the uncertainty of the reconstruction.

\subsection{General Description}

This work describes a navigation algorithm for reconstruction of 3D environments. This algorithm has for input the actual position of the robot and the current reconstruction of the environment. The result is a proposed next position for the robots. 
This position is obtained using the information of the previous reconstruction and its intention is to improve the actual reconstruction.

There are some constraints to this approach, ones come from the robot and sensors capabilities, and others come from the environment, such us occlusion and obstacles. These constraints hinder the computation of an optimal position. An adequate formulation of these constraints is essential for the convergence of the algorithm.

This paper will present the uncertainty model in section 2; this part explains the uncertainty propagation of the pin-hole model. Section 3 covers a simplified occlusion model for structured environments. Section 4 describes the complete navigation algorithm. Finally section 5 and 6 resume the results and future developments for this work.

\section{Uncertainty Model}

The aim of this section is calculating the uncertainty of the $3 \mathrm{D}$ reconstruction of a scene for a given position of a team of robots. Reconstruction model is based on Pinhole projective model. This problem can be solved analytically, but also admits simplifications that reduce computational costs. These simplifications are critical in a recursive problem like this, where uncertainty is calculated on each iteration of the minimization algorithm. The problem has been simplified for team of two robots while maintaining the approach general enough to be extensible to bigger teams.

\subsection{Projective Model Based on Pinhole Model}

Pinhole model is a perspective projection model for 3D objects. Pinhole equations, described in (1), transform a $3 \mathrm{D}$ point $\left(X_{W}, Y_{W}, Z_{W}\right)$ in a $2 \mathrm{D}$ point in the image plane $(u, v)$.The expression (1) assumed that the origin of coordinates in the image plane is at the principal point.

$$
\left[\begin{array}{c}
n u \\
n v \\
n
\end{array}\right]=\left[\begin{array}{ccc}
f_{x} & 0 & 0 \\
0 & f_{y} & 0 \\
0 & 0 & 1
\end{array}\right] \cdot\left[\begin{array}{ll}
R & T
\end{array}\right] \cdot\left[\begin{array}{c}
X w \\
Y w \\
Z w \\
1
\end{array}\right]
$$

Three constraints are considered to solve the equation system.

- Robot movement is restricted to xz plane.

- Focal distance is equal in both axes $f=f_{x}=f_{y}$.

- Optical axes are parallel between them

Formulas in left column are the projective equations for a system of two robots (right \& left), where left robot is situated in $(0,0)$ position and right robot is situated in $\left(\mathrm{t}_{\mathrm{x}}, \mathrm{t}_{\mathrm{z}}\right)$ and $\theta$ orientation. Reconstruction equations (right column) are obtained solving these equations. 
Table 1. Projective and 3D reconstruction equations

\begin{tabular}{|l|l|}
\hline Projective equations & Reconstruction equations \\
\hline$u_{l}=f_{l} \cdot X_{W} / Z_{W}$ & $X_{W}=u_{l} \cdot Z_{W} / f_{l}$ \\
$v_{l}=f_{l} \cdot Y_{W} / Z_{W}$ & $Y_{W}=v_{l} \cdot Z_{W} / f_{l}$ \\
$u_{r}=\frac{f_{r} \cdot\left(\cos \theta \cdot X_{W}+\sin \theta \cdot Z_{W}+t_{x}\right)}{\left(-\sin \theta \cdot X_{W}+\cos \theta \cdot Z_{W}+t_{z}\right)}$ & $Z_{W}=\frac{\left(u_{r} \cdot t_{z}-f_{r} \cdot t_{x}\right) \cdot f_{l}}{\left(f_{r} \cdot \sin \theta-u_{r} \cdot \cos \theta\right) \cdot f_{l}+\left(u_{r} \cdot \sin \theta+f_{r} \cdot \cos \theta\right) \cdot u_{l}}$ \\
$v_{r}=\frac{}{\left(-\sin \theta \cdot X_{W}+\cos \theta \cdot Z_{W}+t_{z}\right)}$ & \\
\hline
\end{tabular}

\subsection{Uncertainty Propagation}

Uncertainty propagation quantifies the effect of variables uncertainties on the uncertainty of a function. In this system, the uncertainty propagation problem deals with estimation of the reconstruction uncertainty given the sensor uncertainty.

Analytical solution. 3D reconstruction uncertainty propagation in correlated variables is calculated through the reconstruction Jacobian matrix $J$ and the sensor covariance matrix $S_{\text {cam }}$, using the formula (2).

$$
S_{W}=[J] \cdot\left[S_{C a m}\right] \cdot[J]^{T}
$$

The Jacobian matrix (3) is defined using the equations defined in table 1 . The sensor covariance matrix is defined by the measurement error in the camera axis (3).

$$
J=\left[\begin{array}{lll}
\frac{d f_{x}\left(u_{r}, u_{l}, v_{l}\right)}{d u_{r}} & \frac{d f_{x}\left(u_{r}, u_{l}, v_{l}\right)}{d u_{l}} & \frac{d f_{x}\left(u_{r}, u_{l}, v_{l}\right)}{d v_{l}} \\
\frac{d f_{y}\left(u_{r}, u_{l}, v_{l}\right)}{d u_{r}} & \frac{d f_{y}\left(u_{r}, u_{l}, v_{l}\right)}{\left.d u_{l}\right)} & \frac{d f_{y}\left(u_{r}, u_{l}, v_{l}\right)}{d v_{l}} \\
\frac{d f_{z}\left(u_{r}, u_{l}, v_{l}\right)}{d u_{r}} & \frac{d f_{z}\left(u_{r}, u_{l}, v_{l}\right)}{d u_{l}} & \frac{d f_{z}\left(u_{r}, u_{l}, v_{l}\right)}{d v_{l}}
\end{array}\right] \quad S_{C a m}=\left[\begin{array}{ccc}
\Delta u_{r} & 0 & 0 \\
0 & \Delta u_{l} & 0 \\
0 & 0 & \Delta v_{l}
\end{array}\right]
$$

Reconstruction error is determined using $S_{W}$, by means of formula (1). Matrix eigenvalues symbolize the uncertainty ellipse axis, an ellipse centred on the threedimensional point that represents the possible values in the reconstruction due to an error in the measurement.

Due to the matrix property whereby the absolute value of all eigenvalues is less than or equal to the norm of the matrix, it can be used the norm of the matrix as an estimation of the uncertainty in the reconstruction. There are different norms that can be used, but the most interesting one is the trace norm, defined in (4).

$$
\|\mathrm{A}\|_{t r}=\operatorname{trace}(\sqrt{\mathrm{A} * \mathrm{~A}})
$$

The analytical solution implies a high computational cost, because of the Jacobian matrix calculation.

Simplified solution. Simplified model is based in a simplification of the Jacobian matrix calculation using the simplification (5) for the derivatives. 


$$
\frac{d f\left(x_{1}, x_{2}, \ldots, x_{i .}, \ldots x_{n}\right)}{d x_{i}} \approx \frac{f\left(x_{1}, x_{2}, \ldots, x_{i .}+\Delta x_{i}, \ldots x_{n}\right)-f\left(x_{1}, x_{2}, \ldots, x_{i,}, \ldots x_{n}\right)}{\Delta x_{i}}
$$

Then, the Jacobian matrix is defined as follows (6).

$$
J=\left[\begin{array}{lll}
\frac{f_{x}\left(u_{r}+\Delta u_{r}\right)-f_{x}\left(u_{r}\right)}{\Delta u_{r}} & \frac{f_{x}\left(v_{r}+\Delta v_{r}\right)-f_{x}\left(v_{r}\right)}{\Delta v_{r}} & \frac{f_{x}\left(u_{t}+\Delta u_{t}\right)-f_{x}\left(u_{t}\right)}{\Delta u_{t}} \\
\frac{f_{y}\left(u_{r}+\Delta u_{r}\right)-f_{y}\left(u_{r}\right)}{\Delta u_{r}} & \frac{f_{y}\left(v_{r}+\Delta v_{r}\right)-f_{y}\left(v_{r}\right)}{\Delta v_{r}} & \frac{f_{y}\left(u_{t}+\Delta u_{t}\right)-f_{y}\left(u_{t}\right)}{\Delta u_{t}} \\
\frac{f_{z}\left(u_{r}+\Delta u_{r}\right)-f_{z}\left(u_{r}\right)}{\Delta u_{r}} & \frac{f_{z}\left(v_{r}+\Delta v_{r}\right)-f_{z}\left(v_{r}\right)}{\Delta v_{r}} & \frac{f_{z}\left(u_{t}+\Delta u_{t}\right)-f_{z}\left(u_{t}\right)}{\Delta u_{t}}
\end{array}\right]
$$

Assuming $\Delta u_{r}=\Delta v_{r}=\Delta u_{l}=1$, sensor covariance matrix is the identity matrix. Then, $S_{W}$ is obtained replacing $J$ and $S_{c a m}$ in the formula (2). Uncertainty estimation is defined as the trace norm of $S_{W}$, that is, as the sum of the square of the Jacobian matrix components (7).

$$
C_{i}=\left\|S_{w}\right\|_{\text {trace }}=\sum_{i=1}^{3} \sum_{k=1}^{3}\left(j_{i k}\right)^{2}
$$
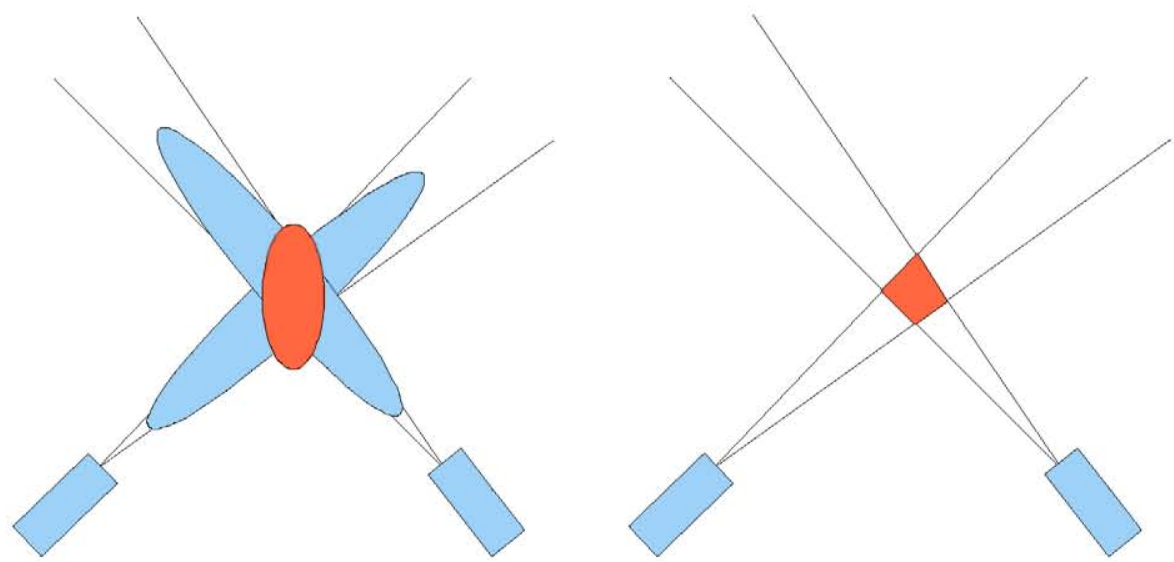

Fig. 1. 3D reconstruction uncertainty in the analytical model and in the simplified model

\section{Occlusion Model}

Given a three dimensional scene, the minimum uncertainty position could have several features occluded by other object of the scene. To solve this problem, the occlusion must be considered in the minimization algorithm. This section describes an occlusion model that determines if an object that is viewed in the original position will be still viewed in the next position.

For this model the following constraints are considered:

- $\quad 3 \mathrm{D}$ scene is defined by a group of points and lines.

- $\quad$ Planes are bounded by lines. 
- Robot only moves on the xy plane.

- Points originally viewed are an input to the system.

The aim is to determine, for all the points in the scene, if they will be occluded by a plane. Assuming that the planes are bounded by lines, a viewed point becomes occluded if the projection of the line crosses over the projection of the point. If the robot moves in the xy plane, the position where the robot can't see the point defines a semiplane. This semi-plane is defined by two points: one is the position where the projection of the line is over the projection of the point and the other is the position of the point we want to see.

At the beginning of the step before the optimization starts, for each possible pair point-line, the semi-plane of occluded positions is calculated. The parameters of all semi-planes are stored in a table. This table is used by the minimization algorithm to determinate if a point is occluded for any position of the robot. Positions of the robot are evaluated as a function of the distance to the bound of the semi-plane. To sum up the algorithm has three steps: determination of the occlusion semi-plane, generation of the occlusion parameters tables and determination of the distance of the robot to the bound of the semi-plane.

The determination of the occlusion plane has as inputs the coordinates of the scene point, the coordinates of the line and the initial position of the robot. First the movement of the robot in a long a circumference arc is simulated. For each position in this trajectory the projections of the point and the line are calculated. Finally, the point in which the projected feature crosses over the projected line is determined. From the coordinates of the robot at this point and the coordinates of the feature the parameters of a semi-plane are estimated.

The parameters of the semi-plane are stored in the form of the two parameters of the equation of a line, the boundary of the semi-plane, and another parameter that determines which side of the semi-plane is occluded and which is not. These three parameters are stored in a matrix for each combination of feature and line visible in the scene.

Inside the minimization algorithm, a given position must be evaluated in relation to its possible occlusions. The value used to evaluate this is the distance from the robot coordinates to the boundary of the occlusion semi-plane. This distance is signed to represent if the robot lays on the occluded side or in the opposite.

This algorithm is useful in structured environments where planes have straight borders, for example walls and furniture. The algorithm presented strongly simplifies the problem of occlusion in favor of the computational costs. On the other hand the algorithm does not consider important cases like holes in the planes or thin columns. Anyway covers a good set of situations in structured environments without a strong computational cost.

\section{Minimization of the Uncertainty and Occlusion Combined Function}

The final aim of the algorithm is to calculate the next position of the robot so the 3D reconstruction of the environment has less uncertainty. The approach presented here uses an optimization algorithm that minimizes the uncertainty. The fundamental 
problem of this work is to find a cost function which minimizes the uncertainty with low computational cost. In addition to this, a number of constraints must be added to the cost function. These constraints cover the limited size of the sensor, the projection over only one side of the sensor, and the environment scale among others.

The resultant cost function is the result of the weighted addition of partial function costs. The cost function will have the form (8)

$$
C=\sum_{\forall P}\left(k_{i} \cdot C_{i}+k_{\alpha} \cdot C_{\alpha}+k_{d} \cdot C_{d}+k_{o c} \cdot C_{o c}\right)
$$

with

- $\quad C_{i}$ Uncertainty minimization

- $C_{\alpha}$ Orientation error minimization

- $\quad C_{d}$ Distance feature-camera

- $C_{o c}$ Occlusion error minimization

The partial cost function for uncertainty minimization is given by the components of the covariance matrix defined in section 2 . The function is defined by the trace norm of the matrix $S_{W}$, defined in (7).

The orientation constraint represents the requirement that the projected point lies inside of the sensor size. This is evaluated minimizing the square of the distance to the projection axis of the camera (9).

$$
C_{\alpha}=u_{l}^{2}+v_{l}^{2}+u_{r}^{2}+v_{r}^{2}
$$

Distance function is used to cover two constraints. The first is that in a real camera de projection plane is only sensitive on one side. Pin-Hole model alone does not restrict the projection of the rays from the back of the sensor. The second constraint is that the camera has a maximum resolution therefore its necessary to control the distance from the feature to the point.

Occlusion constraint considers the visibility of the features. This function weight the distance obtained in section 3 so that is heavily punished when the robot lays in a occluded area. When the robot is outside of the occluded area the function is only slightly punished to keep the distance to the constraint.

This equation fulfills the requirements presented at the beginning of this section. It presents low computational costs, minimizes uncertainty and takes in account the restriction of the sensor and occlusions. The algorithm presented can be extended to include other constraints.

\section{Results}

In this section result of the algorithm and conclusions are analyzed. Fig. 2, Fig. 3, Fig. 4 and Fig. 5 are a representation of the application of the algorithm to a system of two robots. The initial scene is represented in Fig 2 , and contains two view point and an occlusion line. In the following figures can be seen the final position reached by the second robot when the constraints described in each cell. 


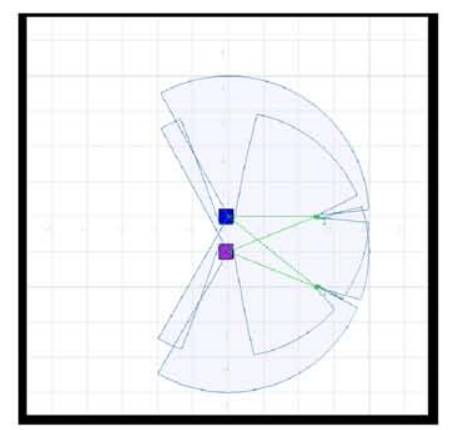

Fig. 2. Initial position

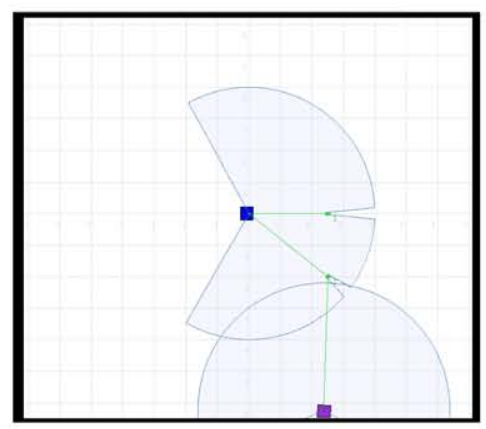

Fig. 4. Final position considering uncertainty and position error

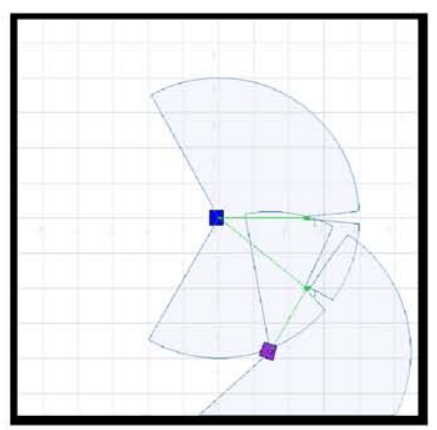

Fig. 3. Final position considering uncertainty error

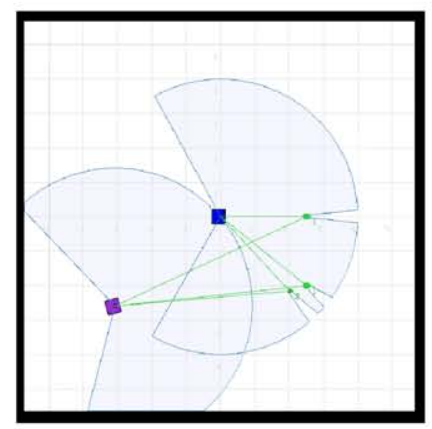

Fig. 5. Final position considering uncertainty, position, distance and occlusion error

In this work a navigation algorithm has been developed. This algorithm uses the $3 \mathrm{D}$ reconstruction uncertainty estimation and the occlusion constraints in the scene. This method has been tested in different scenes with successful results. The objectives related with computational cost and real time requirements have been accomplished.

This work is part of a research project with the name, "Teleoperation architectures in modelling dynamical environments" (DPI 2004-07433-C02-02), in which "Ministerio de Ciencia y Tecnología"(science and technology department) provides economical resources for its development

\section{References}

1. Winchel, W.: Inspection and Measuring in Manufacturing. Keys to process planning and improvement. Society of Manufacturing Engineering (1996)

2. García, D., Sebastián, J.M., Sánchez, F.M., Jiménez, L.M., González, J.M.: 3D inspection system for manufactured machine parts. In: Proceedings of SPIE. Machine Vision Applications in Industrial Inspection VII, vol. 3562, pp. 25-29 (1999)

3. Zhou, L.-X.: Evaluation, evolution and optimal manipulation of uncertainties in computer vision. In: ISPRS International Conference on Intelligent Processing Systems (October 1997) 
4. Rivera-Rios, A.H., Shilh, F.-L., Marefat, M.: Stereo camera pose determination with error reduction and tolerance satisfaction for dimensional measurements. In: Proceedings of the 2005 IEEE. International Conference on Robotics and Automation (April 2005)

5. Lim, S.-N., Davis, L.S.: Visibility planning for active Video Collection. In: European Conference on Computer Vision (June 2006)

6. Quian, X., Harding, K.G.: Partitioning positional and normal space for fast occlusion detection. In: Proceedings of DETC 2003. ASME 2003 Design Engineering Technical Conferences and Computers and Information in Engineering Conference (September 2003)

7. Sanchiz, J.M., Fisher, R.B.: A next-best-view algorithm for 3D scene recovery with 5 degrees of freedom. In: British Machine Vision Conference (1999)

8. Reed, M.K., Allen, P.K.: Constraint-based sensor planning for scene modelling. Computational Intelligence in Robotics and Automation (1999)

9. Bourgault, F., Makarenko, A., Williams, S.B., Grocholosky, B., Durrant-Whyte, H.F.: Information based adaptive robotic exploration. In: Proceedings of the 2002 IEEE International Conference on Intelligent Robots and Systems (2002)

10. Stachniss, C.: Exploration and mapping with mobile robots. Phd Dissertation (April 2006)

11. Burgard, W., Stachniss, C., Crisetti, G.: Information Gain-based Exploration Using RaoBlackwellized Filters. In: Proceedings of the 2005 IEEE International Conference on Intelligent Robots and Systems (2005)

12. Sim, G., Dudek, G., Roy, N.: Online control policy optimization for minimizing map uncertainty during exploration. Robotic and Automation, 2004. In: Proceedings ICRA (2004)

13. Navarro-Serment, L.E., Dolan, J.M., Khosla, P.K.: Optimal sensor placement for cooperative distributed vision. In: Proceedings of the 2004 IEEE. International Conference on Robotics and Automation (April 2004)

14. Mourikis, A.I., Roumeliotis, S.I.: Performance analisys of multi-robot cooperative system. IEEE Transactions on Robotics (August 2006)

15. Göhring, D., Burkhard, H.-D.: Multi-robot object tracking and self localization using visual percept relations. In: Proceedings of the 2006 IEEE. International Conference on Intelligent Robots and Systems (October 2006)

16. Ko, J., Stewart, B., Fox, D., Konolige, K., Limketkai, B.: A practical decision-theoretic approach to multi-robot mapping and exploration. In: Proceedings of the 2003 IEEE. International Conference on Intelligent Robots and Systems (2003)

17. Fox, D., Ko, J., Konolige, K., Limketkai, B.: Distributed multi-robot exploration and mapping. In: Proceedings of the 2006 IEEE (2006) 
Palaniappan, Kannappan 619

Palomo, E.J. 151

Pari, Lizardo 706,718

Passalis, G. 336

Paulhac, Ludovic $\quad 670$

Paz, Juan 221

Penedo, M.G. 122, 807, 1023

Pérez, Marlen 221

Pereira, Fernando 11

Perrotte, S. 385

Pham, Tuan 844

Philips, Wilfried 817

Piccoli, Benedetto 23

Pinho, Cátia 369

Pinto, J.R. Caldas 1041

Pižurica, Aleksandra 817

Pla, Filiberto $\quad 660,1091$

Pokrajac, Dragoljub 680

Pralow, Thomas 854

Quelhas, Pedro 739

Rahmati, Mohammad 966

Ramel, Jean-Yves 670

Regentova, E.E. $\quad 837$

Ribeiro, Eraldo 650

Ribeiro, Miguel 110

Riccio, Daniel 1033

Rizvandi, Nikzad Babaii 817

Robidoux, Nicolas 85

Rocha, Rui 760

Rokita, Joanna 1013

Roli, Fabio 884

Ronsin, Joseph 547

Rosenstiel, Marcus 305

Rouco, J. 122

Roy, Kaushik 894

Rúa, Enrique Argones 1003

Saidane, Zohra 557

Salah-ELDin, Ahmed 873

Sanches, João M. 789

Sánchez, Lidia 827

Sanniti di Baja, Gabriella 132, 507

Sanromà, Gerard 475

Santos, J. 807

Sappa, Angel D.

315

Sav, Sorin 485

Schelkens, Peter 221

Schlüter, Mathias 854
Schulz, Simon 305

Sebastián, José M. 395, 706, 718

Seetharaman, Guna 619

Sequeira, J. 385

Serino, Luca 507

Serrat, Joan 315

Serratosa, Francesc 475

Sgouros, N.P. $\quad 336,379$

Silva, Jorge 760

Smith, William A.P. $\quad 925,955$

Song, H.Y. 281

Soria, Carlos 718

Sotoca, José M. 1091

Sousa, António V. 413

Sousa, J.M.C. 1041

Suarez, Sir 1101

Suen, C.Y. 1013

Sun, Shuwei 204

Sun, Xiaoshuai 587

Tajima, Shigeru 326

Tariq, Anam 915

Teixeira, A.R. 404

Theofanous, N. 379

Theoharis, T. 336

Thériault, J. 779

Tomé, A.M. 404

Toudjeu, Ignace Tchangou 640

Tousignant, Annie 85

Tran, Dat 844

Traslosheros, A. 706, 718

Tsurumi, Satoshi 192

Turcotte, Adam 85

Veni, G. $\quad 837$

Vergés-Llahí, Jaume 294

Vitulano, Domenico $\quad 23,33$

Vitulano, Sergio 863

Vrscay, Edward R. 43, 170, 192

Vural, Fatos Tunay Yarman 445

Wada, Toshikazu 294

Wang, Yue 798

Wang, Zhaozhong 1061

Weickert, Joachim 630

Whelan, Paul F. 688

Wilscy, M. 65

Wu, Jing 925

Wu, Qingrong 798

Wyk, Barend Jacobus van 640

Wyk, Michaël Antonie van 640 